\title{
Intrathoracic extramedullary hematopoiesis presenting as tumor-simulating lesions of the mediastinum in $\alpha$-thalassemia: A case report
}

\author{
JUN AN, YIMIN WENG, JINYUAN HE，YUN LI，SHAOHONG HUANG, \\ SONGWANG CAI and JUNHANG ZHANG \\ Department of Cardiothoracic Surgery, The Third Affiliated Hospital, Sun Yat-Sen University, \\ Guangzhou, Guangdong 510630, P.R. China
}

Received August 22, 2014; Accepted May 8, 2015

DOI: $10.3892 / \mathrm{ol} .2015 .3597$

\begin{abstract}
Extramedullary hematopoiesis (EMH) is a rare disease, where hematological disorder drives extramedullary hematopoietic tumor formation in multiple regions of the body. The present study reports a case of EMH presenting as multiple tumor-like lesions of mediastinum in a 61-year-old male with $\alpha$-thalassemia, which was subjected to a video-assisted thoracoscopic surgery tissue biopsy to differentiate it from other mediastinal tumors. To date, only three cases of EMH in patients with $\alpha$-thalassemia have been described in the literature. Patients with EMH typically exhibit no hematological disorder preoperatively and therefore EMH is frequently misdiagnosed. In the present study, along with a literature review of the clinicopathological features of EMH, the diagnosis and treatment of this rare case was discussed, in order to differentiate diagnosis, and particularly to distinguish EHM from extramedullary myeloid sarcoma.
\end{abstract}

\section{Introduction}

Extramedullary hematopoiesis (EMH), which refers to hematopoiesis occurring outside the medulla of the bone, is a rare hematological disease, secondary to insufficient bone marrow function $(1,2)$. EMH occurs under conditions of myelofibrosis and ineffective erythropoiesis, including that in thalassemia, hereditary spherocytosis and sickle cell disease (3-5). While EMH may occur anywhere in the body, it most commonly

Correspondence to: Professor Junhang Zhang, Department of Cardiothoracic Surgery, The Third Affiliated Hospital, Sun Yat-Sen University, 600 Tianhe Road, Guangzhou, Guangdong 510630, P.R. China

E-mail: zhangjh33@mail.sysu.edu.cn

Key words: mediastinal tumor, extramedullary hematopoiesis, thalassemia presents as diffuse lesions in the liver, spleen and/or lymph nodes. In rare cases, EMH presents as a solitary mass (6), appearing as a tumor-simulating lesion in an atypical location, and is frequently misdiagnosed.

Alpha-thalassaemia is inherited as an autosomal recessive disorder characterized by a microcytic hypochromic anaemia, and is a result of impaired production of $1,2,3$, or 4 alpha globin chains, leading to a relative excess of beta globin chains of haemoglobin $(\mathrm{Hb})(7)$. It is one of the most common monogenic gene disorders worldwide and is particularly frequently observed in Mediterranean countries, South-East Asia and Africa. As a compensatory response in various anaemias, EMH may develop with thalassaemia (7).

The present study describes a case of EMH presenting as multiple tumor-like lesions in the mediastinum of a patient with $\alpha$-thalassemia. The patient was diagnosed with mediastinal EMH following a tissue biopsy via video-assisted thoracoscopic surgery (VATS). The study protocol was approved by the Research Ethics Committee of The Third Affiliated Hospital, Sun Yat-Sen University (Guangzhou, China).

The present case report discusses the major diagnostic problems of mediastinal EMH, distinct characteristics for differentiating EMH from other mediastinal tumors and the treatment strategy applied, as well as a literature review of this rare clinical entity.

\section{Case report}

A 61-year-old Chinese male presented with a 3-year history of intermittent chest pain and tightness. Physical examination revealed elevated blood pressure $(149 / 96 \mathrm{~mm} \mathrm{Hg})$, but no other remarkable findings. A chest X-ray and computed tomography (CT) scan were performed and multiple paravertebral masses were identified (Fig. 1A). Subsequently, a contrast-enhanced CT scan of the chest revealed multiple bilateral mediastinal masses, observed as round or oval, smooth, soft-tissue opacities immediately lateral to the thoracic vertebra. The mass in the left thoracic paravertebral space at the T3-T4 vertebral level measured $44 \times 25 \mathrm{~mm}$, and 
A

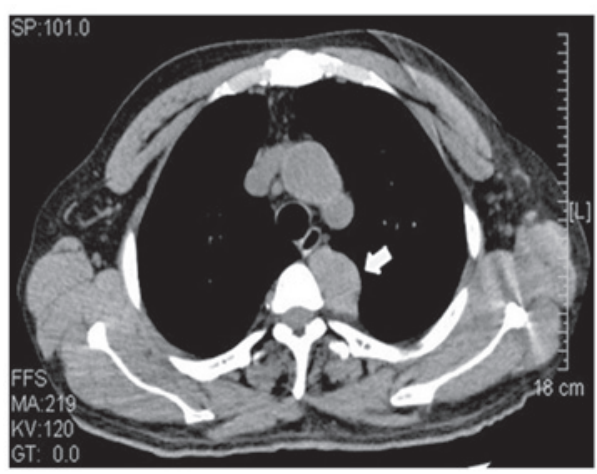

B

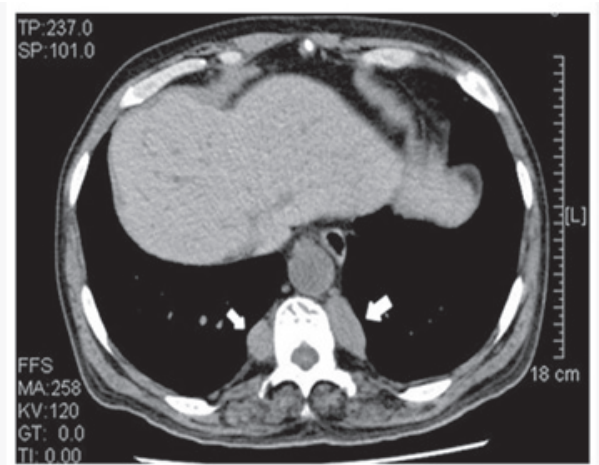

Figure 1. Extramedullary hematopoiesis appears as a solid homogeneous mass (green arrow) in the posterior mediastinal on a thoracic computed tomography scan. (A) T3-4 vertebral level. (B) T10 vertebral level.

the two masses on the right of the T10 vertebra measured $52 \times 48 \times 17 \mathrm{~mm}$ and $32 \times 22 \mathrm{~mm}$ (Fig. 1B). In addition, the initial CT scan results indicated the possibility of neurogenic tumors.

The complete blood count revealed the following results: White blood cells, 11.44×109/1; hemoglobin (Hb), $119 \mathrm{~g} / \mathrm{l}$; platelets, 176×10\%/1; mean corpuscular volume, $76.4 \mathrm{fl}$; mean corpuscular Hb concentration, $258 \mathrm{~g} / \mathrm{l}$; segmental neutrophils, 48.1\%; lymphocytes, $44.5 \%$; monocytes, $5 \%$ and eosinophils, $1.9 \%$. No pathological changes were detected in erythrocyte sedimentation rate or C-reactive protein.

To determine the diagnosis of the mediastinal masses, a surgical tissue biopsy via VATS was performed following the provision of consent of the patient and family. A section of the dark-red mass in the left thoracic cavity was excised and electrocoagulation was used for hemostasis. Frozen section examination revealed EMH. A conventional permanent histopathological examination was also performed; the biopsied tissue was stained (hematoxylin and eosin) and examined under a BX43 microscope (Olympus Corporation, Tokyo, Japan), revealing hyperplasia with activity of two types of hematopoietic cell, namely erythroid and megakaryocytic cells (Fig. 2). Furthermore, immunohistochemical staining indicated that diffuse cells from the mediastinal lesions were positive for CD43, CD99 and high Ki-67, while sporadic cells were positive for myeloperoxidase, CD15, CD3, CD45RO and CD61. The diffuse and sporadic cells were negative for creatine kinase, CD30 and Pax-5. Myeloid sarcoma, a rare extramedullary form of myelogenous leukemia, also known as granulocytic sarcoma or chloroma was therefore excluded. Based on these pathological and clinical findings, the patient was diagnosed with thoracic EMH.

Postoperatively, various laboratory tests were performed, which demonstrated low $\mathrm{Hb}$ (90 g/l). Subsequently, thalassemia-associated tests were performed. A diagnosis of $\alpha$-thalassemia was made based on the increased glucose- 6 -phosphate dehydrogenase activity $(15919.000 \mathrm{mU} / \mathrm{g})$, decreased HbA2 (0.9\%) and RBC-BT (erythrocyte fragility test; $29.0 \%$ ), as well as positive hemoglobin $\mathrm{H}$ staining with hemoglobin electrophoretic studies ( $\mathrm{HbH}, 10.7 \%)$.

The patient was transferred to the Department of Hematology for further treatment, where bone marrow aspiration cytology confirmed the findings of anemia and erythroid hyperplasia (G:E, 0.82:1). In addition, the relative proportion

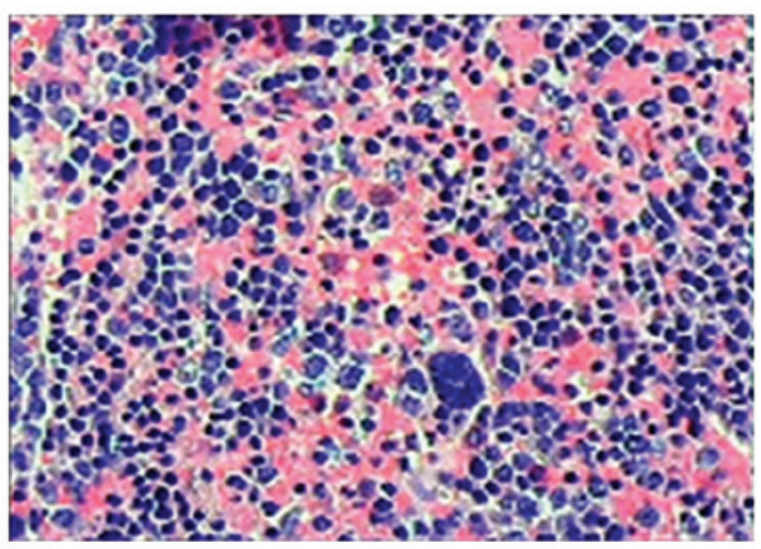

Figure 2. EMH microscopic findings. A focus of EMH, characterized by the presence of hematopoietic cells, erythrocytes and megakaryocytes, in the resected specimen (hematoxylin and eosin stain; magnification, x100). EMH, extramedullary hematopoiesis.

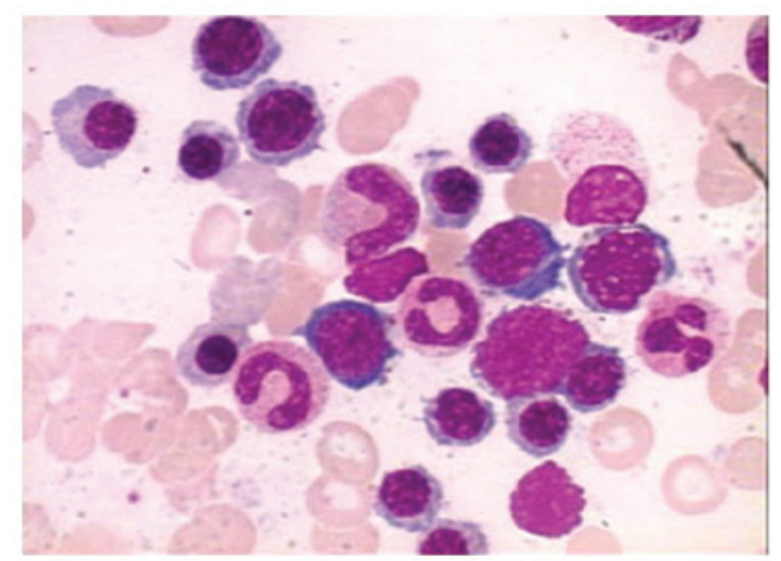

Figure 3. Bone marrow aspirate analysis. A bone marrow aspirate revealed erythroid hyperplasia (G:E, $0.82: 1$ ), and the relative proportion of immature red blood cells was also increased but no malignant cell invasion was observed. (Wright-Giemsa stain; magnification, x1,000).

of immature red blood cells was also significantly increased, but was not accompanied by malignant cell invasion (Fig. 3).

Subsequently, 18-fluorodeoxyglucose (FDG) positron emission tomography-computed tomography (PET/CT) was performed to evaluate the mediastinal masses and determine 
A

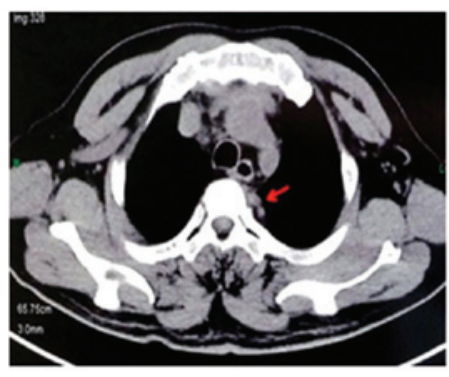

D

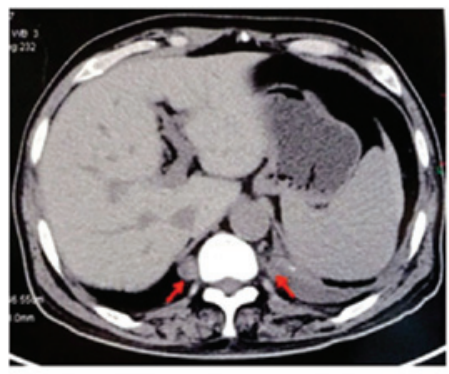

B

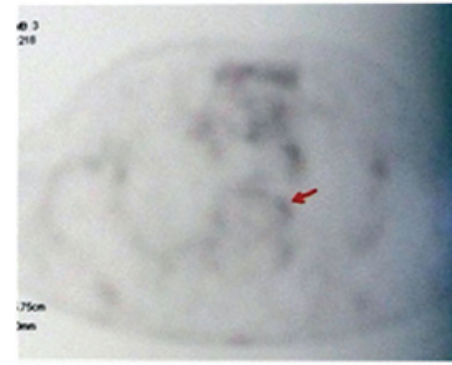

E

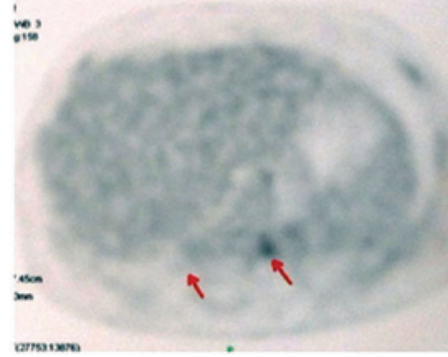

C

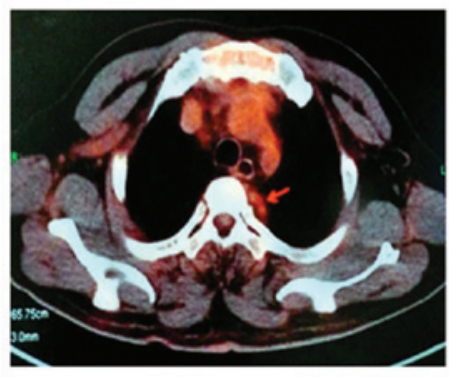

$\mathbf{F}$

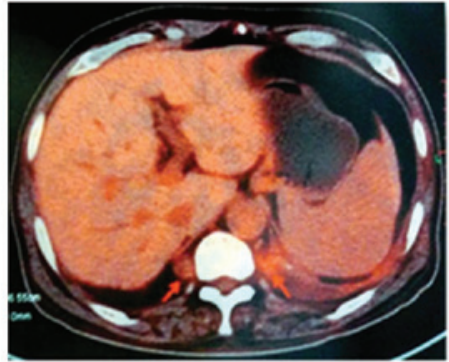

Figure 4. EMH at the posterior-superior mediastinum (T3-T4 vertebral level) and the posterior-inferior mediastinum (T10 vertebral level) on a postoperative PET/CT scan (red arrow). (A) CT image, (B) PET image and (C) fused image at T3-T4 vertebral level. PET/CT indicated slightly increased FDG uptake in mediastinal mass at the T3-T4 vertebral level. ( $\mathrm{SUV}_{\max }, 5.2$; SUV average, 2.8). (D) CT image, (E) PET image and (F) fused PET/CT image at the T10 vertebral level detected EMH as a benign-appearing mass. $\mathrm{SUV}_{\max }$ was markedly decreased in EMH (SUV $\max , 3.8$; SUV average, 2.4). EMH, extramedullary hematopoiesis; PET/CT, positron emission tomography-computed tomography; FDG, 18-fluorodeoxyglucose.

the presence of any additional extramedullary masses. PET/CT identified remnant tissue in the left thoracic T3-T4 paravertebral space following biopsy, and the maximum standardized uptake value $\left(\mathrm{SUV}_{\max }\right)$ was 5.2. The remaining two paravertebral masses, at the posterior-inferior mediastinum at the T10 level, demonstrated no increased glucose metabolism, with $\mathrm{SUV}_{\max }$ values of 3.8 (Fig. 4).

Since the postoperative course following VATS was uneventful and the patient exhibited no clinical symptoms, regular outpatient follow-up observation was selected, rather than treatment. At 8 months postoperatively, at the most recent follow-up visit, the patient exhibited no evidence of disease.

\section{Discussion}

For a primary mediastinal mass, the potential clinical differential diagnoses are extensive. However, EMH, a rare entity involving the formation and development of blood cells outside of the bone marrow, is an unlikely diagnostic candidate.

EMH occurs in response to the failure of erythropoiesis in bone marrow and may occur in myeloproliferative disorders, hemoglobinopathies or bone marrow infiltration. EMH most commonly affects the spleen and liver and, occasionally, the lymph nodes. Less commonly involved organs include the pleura, lungs, gastrointestinal tract, breast, skin, brain, kidneys and adrenal glands (8). The occurrence of EMH as a tumor-simulating mass in the intrathoracic cavity, particularly a posterior mediastinal mass, is very rare (9). A review of the literature revealed that only three cases of EMH in patients with $\alpha$-thalassemia have been reported, to date $(5,10,11)$.

$\mathrm{EMH}$ is frequently characterized by the development of soft tissue masses in the paravertebral thoracic regions. These masses rarely induce significant symptoms, but may result in hemothorax and pleural effusion $(5,12)$. In the present case, the patient was asymptomatic and EMH was identified by chance.
Patients with no hematologic disorder, presenting with such masses induced by EMH, are frequently misdiagnosed $(9,13)$. EMH is often incidentally diagnosed, when patients undergo evaluation for unrelated symptoms, as in the present case. While EMH diagnosis requires a biopsy, a needle biopsy carries the risk of hemothorax. In the presence of anemia, a diagnosis of EMH may be considered preoperatively; however, in the present case, the primary blood tests identified $\mathrm{Hb}$ as $119 \mathrm{~g} / \mathrm{l}$; therefore, EMH was initially overlooked as a potential diagnosis.

Notably, extramedullary myeloid sarcoma (EMS) is also a rare extra medullary tumor mass consisting of myeloid blasts. EMS is also known as granulocytic sarcoma or chloroma, since it is diagnosed based on the presence of a green color caused by myeloperoxidase. EMS occurs in $~ 5 \%$ of patients with acute myeloid leukemia (AML) (14). Rarely, EMS may predate AML, presenting as an isolated mass in various sites of the body. However, EMS in the mediastinal region has rarely been reported $(15,16)$. EMS is primarily diagnosed based on immunohistochemical staining of tissue biopsy. Although EMH and EMS arise from distinct hematological diseases, the two conditions should be considered as differential diagnoses for a mediastinal tumor.

A PET/CT scan may be diagnostically useful in such cases. To date, only a few cases regarding the diagnosis of EMH using PET/CT have been reported $(17,18)$, with EMH detected as a benign mass with low $\mathrm{SUV}_{\max }$ values and normal appearance of the tissue. The concurrent presence of an underlying hematopoietic disorder may suggest a diagnosis of EMH. In EMS, a PET/CT scan identifies intense FDG uptake (16).

As a result of its rarity, there are no evidence-based guidelines for the treatment of thoracic pseudotumors induced by EMH. Essentially, EMH is a compensatory mechanism for chronic anemia; therefore, recurrent blood transfusions to correct such anemia may result in shrinkage of the mass. 
However, blood transfusions are not entirely harmless or risk-free, and this therapeutic effect is temporary, slow to occur and may be insufficient (19-21). Hematopoietic tissue is notably radiosensitive and undergoes shrinkage with radiotherapy; thus, excellent results have been obtained in cases of EMH with a few days of radiotherapy alone $(22,23)$. Surgical excision is required in patients with EMH associated with complications, including bleeding from the mass (9), spinal cord compression or spinal canal invasion (13). Such excision does not only staunch the bleeding and/or allow decompression but also provides a histological diagnosis. Following surgical excision, radiotherapy may aid the prevention of recurrence of EMH or facilitate complete remission $(24,25)$.

Based on the literature review, it was hypothesized that close follow-up of the patient was important. Considering the excellent results obtained with low-dose radiotherapy for EMH masses, the patient will be advised to undergo radiation therapy in the future.

EMH should be considered in the differential diagnosis of an intrathoracic mass. Particularly, if a history of thalassemia or chronic anemia is discovered, based on evaluation of the patient's medical history, as well as laboratory studies. Surgical resection and radiation therapy should be considered as treatment options for EMH.

\section{Acknowledgements}

The present study was supported by the ' 985 ' project grant of Sun Yat-sen University (no. 82000-31101301), which was awarded to Dr Junhang Zhang (The Third Affiliated Hospital, Sun Yat-Sen University, Guangzhou, China).

\section{References}

1. Bolaman Z, Polatli M, Cildag O, Kadikoylu G and Culhaci N: Intrathoracic extramedullary hematopoiesis resembling posterior mediastinal tumor. Am J Med 112: 739-741, 2002.

2. Xiros N, Economopoulos T, Papageorgiou E, Mantzios G and Raptis S: Massive hemothorax due to intrathoracic extramedullary hematopoiesis in a patient with hereditary spherocytosis. Ann Hematol 80: 38-40, 2001.

3. Gumbs R, Ford EAH, Teal JS, Kletter GG and Castro O: Intrathoracic extramedullary hematopoiesis in sickle cell disease. Amer J Radiol 149: 889-893, 1987.

4. Scott L, Blair G, Taylor G, Dimmick J and Fraser G: Inflammatory pseudotumors in children. J Pediatr Surg 23: 755-758, 1988.

5. Chu KA, Lai RS, Lee CH, Lu JY, Chang HC and Chiang HT: Intrathoracic extramedullary haematopoiesis complicated by massive haemothorax in alpha-thalassaemia. Thorax 54: 466-468, 1999.

6. Haidar R, Mhaidli H and Taher AT: Paraspinal extramedullary hematopoiesis in patients with thalassemia intermedia. Eur Spine J 19: 871-878, 2010.

7. Harteveld CL and Higgs DR: Alpha-thalassaemia. Orphanet J Rare Dis 5: 13, 2010.

8. Choi H, David CL, Katz RL and Podoloff DA: Case 69: Extramedullary hematopoiesis. Radiology 231: 52-56, 2004.
9. Kubokura H, Koizumi K, Yoshino N, Yamagishi S, Mikami I, Hirata T, Harada A, Kawamoto M and Shimizu K: A case report: Thoracic extramedullary hematopoiesis found by occurring spontaneous pneumothorax. Ann Thorac Cardiovasc Surg 14: 382-385, 2008

10. Bobylev D, Zhang R, Haverich A and Krueger M: Extramedullary haematopoiesis presented as intrathoracic tumour in a patient with alpha-thalassaemia. J Cardiothorac Surg 8: 120, 2013.

11. Wu JH, Shih LY, Kuo TT and Lan RS: Intrathoracic extramedullary hematopoietic tumor in hemoglobin $\mathrm{H}$ disease. Am J Hematol 41: 285-288, 1992.

12. Peng MJ, Kuo HT and Chang MC: A case of intrathoracic extramedullary hematopoiesis with massive pleural effusion: Successful pleurodesis with intrapleural minocycline. J Formos Med Assoc 93: 445-447, 1994.

13. Yeom SY, Lim JH, Han KN, Kang CH, Park IK and Kim YT: Extramedullary hematopoiesis at the posterior mediastinum in patient with hereditary spherocytosis: A case report. Korean J Thorac Cardiovasc Surg 46: 156-158, 2013.

14. Liu PI, Ishimaru T, McGregor DH, Okada H and Steer A: Autopsy study of granulocytic sarcoma (chloroma) in patients with myelogenous leukemia, Hiroshima-Nagasaki 1949-1969. Cancer 31: 948-955, 1973.

15. Nounou R, Al-Zahrani HH, Ajarim DS, Martin J, Iqbal A, Naufal R, Stuart R, Roberts G and Gyger M: Extramedullary myeloid cell tumours localised to the mediastinum: A rare clinicopathological entity with unique karyotypic features. J Clin Pathol 55: 221-225, 2002.

16. Makis W, Hickeson M and Derbekyan V: Myeloid sarcoma presenting as an anterior mediastinal mass invading the pericardium: Serial imaging with F-18 FDG PET/CT. Clin Nucl Med 35: 706-709, 2010.

17. Zade A, Purandare N, Rangarajan V, Shah S, Agrawal A, Ashish J and Kulkarni M: Noninvasive approaches to diagnose intrathoracic extramedullary hematopoiesis: 18F-FLT PET/CT and 99mTc-SC SPECT/CT scintigraphy. Clin Nucl Med 37: 788-789, 2012.

18. Mosley C, Jacene HA, Holz A, Grand DJ and Wahl RL: Extramedullary hematopoiesis on F-18 FDG PET/CT in a patient with metastatic colon carcinoma. Clin Nucl Med 32: 878-880, 2007.

19. Chehal A, Aoun E, Koussa S, Skoury H, Koussa S and Taher A: Hypertransfusion: A successful method of treatment in thalassemia intermedia patients with spinal cord compression secondary to extramedullary hematopoiesis. Spine (Phila Pa 1976) 28: E245-E249, 2003.

20. Tai SM, Chan JS, Ha SY, Young BW and Chan MS: Successful treatment of spinal cord compression secondary to extramedullary hematopoietic mass by hypertransfusion in a patient with thalassemia major. Pediatr Hematol Oncol 23: 317-321, 2006.

21. Berkmen YM and Zalta BA: Case 126: Extramedullary hematopoiesis. Radiology 245: 905-908, 2007.

22. Singhal S, Sharma S, Dixit S, De S, Chander S, Rath GK and Mehta VS: The role of radiation therapy in the management of spinal cord compression due to extramedullary haematopoiesis in thalassaemia. J Neurol Neurosurg Psychiatry 55: 310-312, 1992.

23. Wen BC, Hussey DH, Hitchon PW, Schelper RL, Vigliotti AP, Doornbos JF and VanGilder JC: The role of radiation therapy in the management of ependymomas of the spinal cord. Int J Radiat Oncol Biol Phys 20: 781-786, 1991.

24. Dore F, Pardini S, Gaviano E, Longinotti M, Bonfigli S, Rovasio S, Tomiselli A and Cossu F: Recurrence of spinal cord compression from extramedullary hematopoiesis in thalassemia intermedia treated with low doses of radiotherapy. Am J Hematol 44: 148, 1993.

25. Neben-Wittich MA, Brown PD and Tefferi A: Successful treatment of severe extremity pain in myelofibrosis with low-dose single-fraction radiation therapy. Am J Hematol 85: 808-810, 2010. 